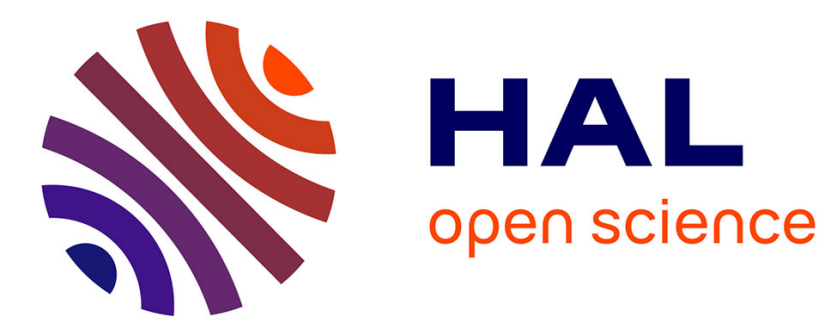

\title{
Subsign detection with region-growing from contrasted seeds
}

\author{
Anne-Sophie Puthon, Fabien Moutarde, Fawzi Nashashibi
}

\section{To cite this version:}

Anne-Sophie Puthon, Fabien Moutarde, Fawzi Nashashibi. Subsign detection with region-growing from contrasted seeds. ITSC, Sep 2012, Anchorage, United States. hal-00741617

\section{HAL Id: hal-00741617 \\ https://hal.inria.fr/hal-00741617}

Submitted on 14 Oct 2012

HAL is a multi-disciplinary open access archive for the deposit and dissemination of scientific research documents, whether they are published or not. The documents may come from teaching and research institutions in France or abroad, or from public or private research centers.
L'archive ouverte pluridisciplinaire HAL, est destinée au dépôt et à la diffusion de documents scientifiques de niveau recherche, publiés ou non, émanant des établissements d'enseignement et de recherche français ou étrangers, des laboratoires publics ou privés. 


\title{
Subsign detection with region-growing from contrasted seeds
}

\author{
Anne-Sophie Puthon, Fabien Moutarde and Fawzi Nashashibi
}

\begin{abstract}
Speed limit determination systems for cars based on vision are more and more developed. Roadsign detection is nowadays a well managed problem. However, in some situations this information is not sufficient to know the speed limitation. Restrictions are sometimes applicable and specified by subsigns. These small rectangles often provide essential information about the applicability scope (vehicle type, condition, lane, etc.) of speed limits. We present an approach of subsign localization based on region growing with an initial step of seed selection using morphological reconstruction. A comparison is also performed with three other techniques based on edge, color and graph on two databases gathering French and German subsigns. The obtained subsign correct detection is above $65 \%$.
\end{abstract}

\section{INTRODUCTION}

With the increasing need of making car a safe mean of transport comes the development of more and more sophisticated ADAS (Advanced Driving Assistance Systems). To help the driver having the most complete overview of its surrounding environment, manufacturers equip their cars with lots of sensors and dedicated systems, like pedestrian detection or lane keeping assistant. Concerning the speed management, TSR (Traffic Sign Recognition) systems aim at detecting and recognizing speed limit signs located on the side (or above) of the road by using an embedded camera. However, in some situations, these limitations are restricted to a particular category of vehicle, circumstance (weather condition, date or time, etc.) or lane (in highway exits). This additional information is specified by supplementary rectangular signs located under the corresponding speed limit. The main challenges of the task are the variability of the signs in size and ratio as well as in the type of information they provide (arrow, text, pictogram, etc.). Figure 1 illustrates some various subsigns encountered on road. Moreover, intelligent vehicle systems require high performance and must work in unsupervised environment with a moving camera.

Unfortunately, nowadays only very few dedicated methods have been implemented for the localization of these subsigns ([1], [2] and [3]). By extension, subsigns can be seen as rectangular homogeneous regions with contrasted symbols. Thus, related subjects are detection of U.S. speed limit signs, license plate or direction indicators localization. They are all

Manuscript received July 12th, 2012.

A.-S.P. and F.M. are from the Robotics Centre of Mines ParisTech, 60 boulevard Saint-Michel F-75272 Paris Cedex 06, FRANCE (phone: (33)140-51-94-54, email: \{anne-sophie.puthon, fabien.moutarde\} @ensmp.fr)

F.N. is both from the Robotics Centre of Mines ParisTech and from INRIA, IMARA Team, BP 105 F-78153 Le Chesnay Cedex, FRANCE (phone: (33)1-39-63-52-56, email: fawzi.nashashibi@inria.fr)
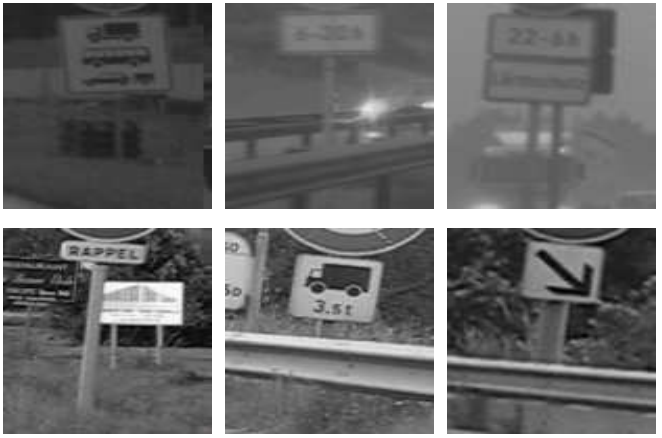

Fig. 1. Example of German (top) and French (bottom) subsigns.

designed for being seen by drivers at a relative great distance, i.e. on clean background with a readable font. Among the different approaches a distinction can be made between edge, color and spatial based methods. Edge-based techniques rely on the border detection and the rectangular shape of the objects. Jung et al. [4] use the Hough transform to retrieve the rectangles in the image with their parameters (size, ratio, orientation). Keller et al. [5] and Hamdoun et al. [1] search for horizontal and vertical lines through a voting scheme based on either a gradient map or a Canny filtered image. Color-based methods perform histogram thresholding [6] based on the assumption that the searched object all contain a significant proportion of uniformly colored background (usually a "dirty" yellowish white or grey depending on illumination and contrast). The last approach of Wu et al. [7] consists in detecting and clustering keypoints in the image. Then groups verifying a vertical plane hypothesis are kept and assume to belong to roadsigns.

Considering that without contrasted foreground no subsign can be detected, we combine a hole search with a region growing approach. The former consists in selecting pixels with a high contrast with the surrounding region while the latter allow us to delineate homogeneous regions. The developed approach is therefore not limited to the subsign detection but is generalizable to the detection of different types of informative regions like the U.S. speed signs. To validate our method we compared it to several techniques using different types of characteristics. The paper is organized as follows. Section II describes our region growing approach. The three comparative algorithms are introduced in section III as well as the evaluation protocol and the results. Finally, section IV presents the conclusion and future works. 


\section{REGION GROWING FROM CONTRASTED SEEDS}

\section{A. Region growing}

Region-based approaches aim at segmenting the image into connected and homogeneous groups of pixels. Introduced by Zucker [8], rgion growing consists in selecting a set of "seeds", or initial regions $R_{0}$, to which neighbouring pixels are agglomerated if they fulfill homogeneity predicates. It is a bottom-up approach contrary to the splitting technique which starts from a set of regions which are iteratively split if the criteria are not verified. The principle is the following:

\section{1) Selection of the seeds}

The first critical issue is the choice of the initial sets of pixels from which regions will grow. They actually need to belong to the objects to segment and must be a representative part of them. In section II-B we introduce our specific method for extracting seeds belonging to the subsign. It is based on a hole search.

\section{2) Selection of candidates for the aggregation}

At each iteration a set of pixels is added to a region $R$ until the equilibrium is reached. To be eligible, a pixel $p$ must be adjacent to the current region $R$ (in the sense of the 4- or 8-connexity) and verify a local homogeneity criterion $\kappa_{L}$ such that:

$$
\exists q \in N_{v}(p) \cap R,\left|\frac{I(p)}{I(q)}-1\right| \leq \kappa_{L}
$$

with $N_{v}(p)$ the set of neighboring pixels of $p$ and $I(p)$ the grey value of pixel $p$ in the image $I$.

\section{3) Agglomeration}

A candidate pixel $p$ is finally merged to the region $R$ if the global homogeneity predicate $\kappa_{G}$ is verified:

$$
\left|\frac{I(p)}{\mu_{R_{0}}}-1\right| \leq \kappa_{G}
$$

where $\mu_{R_{0}}$ is the mean value of the initial region $R_{0}$. This criteria ensures that the global variance of the region is limited and less than $2 \mu_{R_{0}}$.

The values experimentally chosen for our implementation as giving the best results are $N_{v}=4, \kappa_{L}=0.1$ and $\kappa_{R}=0.1$.

\section{B. Selection of seeds}

To draw the drivers' attention, road information are assumed to be highly contrasted, generally black symbols on white background. To segment these local extrema, we use the morphological reconstruction of an image $I$ with marker $M$ of Vincent [9]. Basically, it helps to extract the peaks of the image marked by $M$. This operator can be expressed as:

$$
\rho_{I}(M)=\bigvee_{n \geq 1} \delta_{I}^{(n)}(M)
$$

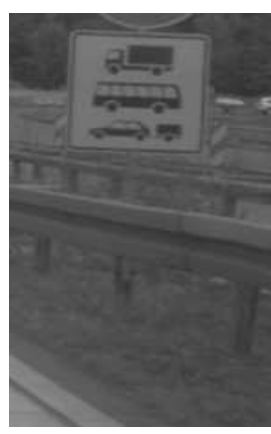

(a) Original image

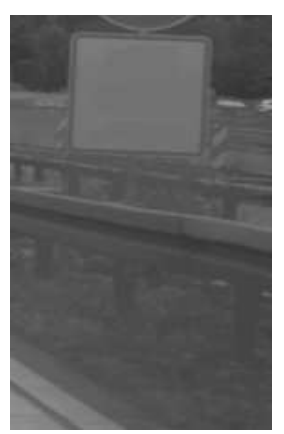

(b) Reconstruction result.

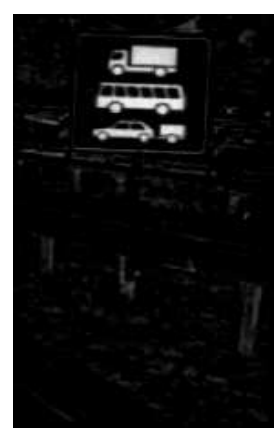

(c) Resulting holes.

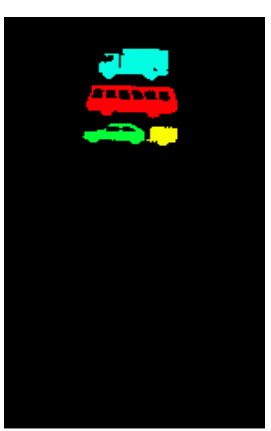

(d) Connected components selected.

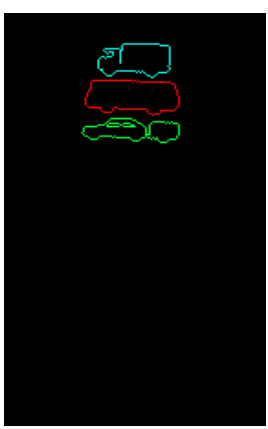

(e) Sets of seeds used for the region growing.
Fig. 2. Illustration of our method of seed selection. The reconstruction 2(a) has filled all the holes of the original image. By subtracting 2(b) from 2(a) we recover the contrasted pixels $2(\mathrm{c})$. Then we proceed to a connected component extraction 2(d) of the thresholded image applying 5. Finally, the seeds used by the region growing correspond to the pixels located at a distance $d=1$ of these components 2(e).

where $\delta_{I}^{(n)}(M)$ is the geodesic dilatation of $M$ of size $n$ and $\bigvee_{n} X(n)$ means the maximum value taken by $X(n)$ for all $n$.

The seed selection procedure is depicted in figure 2 and consists in:

\section{1) Filling the holes}

As we are searching for the holes in the image, we perform the morphological reconstruction of the complemented image $\tilde{I}=255-I$ of the source image $I$. The marker is designed to extract only the connected components which do not touch the image borders.

\section{2) Extracting the holes}

By subtracting the resulting image $\rho_{\tilde{I}}(M)$ from the source $I$, we get the only pixels belonging to the dark connected components surrounded by light ones.

$$
I_{H}=I-\rho_{\tilde{I}}(M)
$$

\section{3) Filtering}

In order to filter out the slightly contrasted pixels, we threshold the image depending on the mean $\mu$ and the standard deviation $\sigma$ of $I_{H}$. The remaining pixels are then grouped into connected components. Only the 
ones containing at least 3 highly contrasted pixels are kept. This ensures to eliminate some noisy elements. Thus, the remaining components $C C$ must verify:

$$
\left\{\begin{array}{l}
\forall p \in C, I_{H}(p) \geq \mu+\sigma \\
\operatorname{Card}\left(p \in C \mid I_{H}(p) \geq \mu+3 \sigma\right) \geq 3
\end{array}\right.
$$

\section{4) Generating the seeds}

In our approach, the regions to grow are the light homogeneous and rectangular subsigns surrounding these dark pixels. We therefore consider all the pixels located at a distance dist $=1$ from the previous black connected components. Each connected set of these pixels represents an initial region for subsigns. By choosing directly a set as seed rather than a single pixel, the process is speeded up and made robuster as more information about the region is available.

\section{Evaluation}

\section{A. A comparative study}

As mentioned earlier only few techniques were implemented for the subsign detection, mainly using edges. To evaluate our approach, we thus implemented an existing one. Moreover we developed two other methods based on image segmentation and relying on different features such as color distribution and spatial relationships. The objectives was to compare our technique of region growing to diverse alternatives.

\section{- Edge-based}

This approach aims at detecting the subsign borders by the use of a Canny filter [1]. It works similarly to a template-matching. Horizontal and vertical lines are first searched for in the image. Then they are grouped by pairs and finally in rectangles if they meet certain conditions.

\section{- Color-based}

We implemented a technique based on the histogram distribution from Zhang et al. [10]. This choice is motivated by the assumption that a subsign and its background must have different color mode to be detected. The objective is to fit the histogram to a mixture of Gaussians without knowing a priori the number of modes. It consists in iteratively splitting and merging the Gaussians previously evaluated as the worst fitting the current estimation.

\section{- Graph-based}

Felzenszwalb et al. [11] proposed a segmentation lying on a compromise between the internal homogeneity of regions Int and the dissimilarity $D i f$ between them. Two regions $R_{1}$ and $R_{2}$ are separable if

$$
\operatorname{Dif}\left(R_{1}, R_{2}\right)>\min \left(\operatorname{Int}\left(R_{1}\right)+\tau\left(R_{1}\right), \operatorname{Int}\left(R_{2}\right)+\tau\left(R_{2}\right)\right.
$$

where $\tau(R)=\kappa /|R|$ is the threshold function. We modify the threshold to take into consideration the rectangular shape of the region and its global homogeneity.

$$
\tau(R)=\frac{\kappa}{|R|}\left(\beta_{\text {size }}+\beta_{\text {rect }} \cdot f_{\text {rect }}(R)+\beta_{\text {hom }} \cdot f_{\text {hom }}(R)\right)
$$

$f_{\text {rect }} \rightarrow 0$ when the shape is close to a rectangle.

$f_{\text {hom }}(R) \rightarrow 0$ when $\operatorname{var}(R) \rightarrow 0$

For every approach, a further step of region filtering is performed. It aims at keeping only those centered in the image, with a ratio, width and height within a given interval.

\section{B. Evaluation procedure}

\section{- Databases}

To our knowledge, no database is currently available for the specific task of subsign detection/recognition. Hence, we compared the four techniques on our own databases. We dispose of subsigns of two countries, France and Germany. This allows us to perform a first evaluation in an European context. Table I gives an overview of the two databases of the study. We consider the total number of subsigns in the sense of a frame per frame evaluation.

\section{- Comparison criteria}

The rectangles output by the different approaches are compared with several criteria. Firstly, in many TSR applications segmented regions are evaluated with the Jaccard's measure $J$ (see figure 3 ).

$$
J=\frac{G T \cap A L G O}{G T \cup A L G O}=\frac{I}{U}
$$

A high value of $J$ shows a good overlapping surface $I$ between the ground truth (GT) and the algorithm result (ALGO) with a low disjoint area. However, the use of this measure only can be misleading. Figure 4 shows three different configurations of Ground Truth and Algorithm giving the same value of $J$. Using this single measure to validate or not a rectangle can result in a loss of information, as the detection is the early stage of the whole system.

To compare our detection techniques, we secondly introduce two other criteria, the overlap $O$ and the centering

\begin{tabular}{|c|c|c|}
\hline Database & Country & Subsigns (fr) \\
\hline$d b F$ & France & 1040 \\
$d b G$ & Germany & 12546 \\
\hline \multicolumn{3}{|c|}{ TABLE I } \\
DATABASE STATISTICS.
\end{tabular}




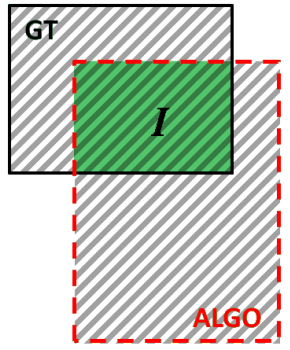

Fig. 3. Illustration of the Jaccard's measure $J$. The intersection $I=$ $G T \cap A L G O$ corresponds to the overlap between the Ground Truth (GT) and the region output by the algorithm (ALGO). $U=G T \cup A L G O$ (in stripes) is the union of both regions.
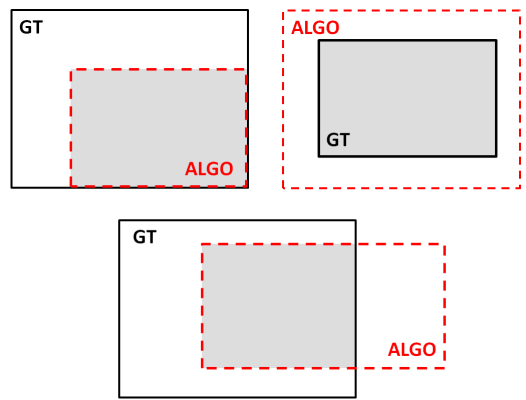

Fig. 4. Examples of three configurations of GT and ALGO resulting in $J=0.5$.

$$
C \text {. }
$$

$$
\begin{aligned}
& O=\frac{I}{\operatorname{Area}(G T)} \\
& D=\frac{A L G O \backslash G T}{\text { Area }(G T)}
\end{aligned}
$$

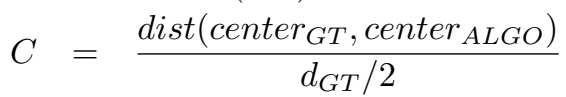

The overlap has an interest in the context of a further classification allowing to control the amount of overlapping area the recognition needs. The last criteria $C$ corresponds to the ratio of the distance between the region centres and the half of the diagonal $d_{G T}$ of GT.

\section{- Results}

The comparison is performed for the criteria values:

$$
\begin{aligned}
& J \geq 0.5 \\
& O \geq 0.5 \\
& O \geq 0.5 \& D \leq 1.5 \\
& C \leq 0.2
\end{aligned}
$$

Results are shown in table II. The Jaccard's measure defined in equation 11 corresponds to the value selected in most of the paper about subsign detection. An overlap of 0.5 ensures that at least the half of the ground truth is covered by the algorithm. Combined to a disjoint measure less than 1.5, output regions are limited and avoid a further classification step to deal with noisy regions.
Generally speaking, the color-based approach appears to give the worst results. An explanation can be that our grayscale source images do not give enough color information to be able to efficiently detect the subsigns. Moreover, the process of splitting and merging until equilibrium is quite long and fastidious making this technique not very applicable as is. The two best techniques are the edge-based and ours regarding $J$ or the combination of $O$ and $D$. Regarding the centering measure, the best technique is the region growing as expected. The contrasted pixels used to generate the initial seeds are indeed mainly located in the middle of subsigns. Figure 7 shows the feature image and the resulting rectangles for each method. Finally, some results obtained with our region-growing technique are given in figures 5 and 6 for French and German subsigns. Actually the main sources of errors come from:

- a lack of contrast, appearing for instance in rainy conditions or when blur moving occurs;

- the absence of seeds in the region growing process because of an insufficient contrast of the symbols.

\section{CONCLUSION AND FUTURE WORKS}

We presented a new technique of subsign detection based on region growing with a search of contrasted pixels as initial seeds. This idea was motivated by the assumption that roadsign in general are specifically designed to be seen by drivers at great distances. We thus implemented an approach based on a morphological reconstruction to get highly contrasted holes in the image. A comparison was then performed with three other image processing-based approaches in order to validate our method. For this evaluation, we proposed three different criteria adapted to the detection task. The final results highlight two algorithms, ours and the modified graph-based, with correct subsign
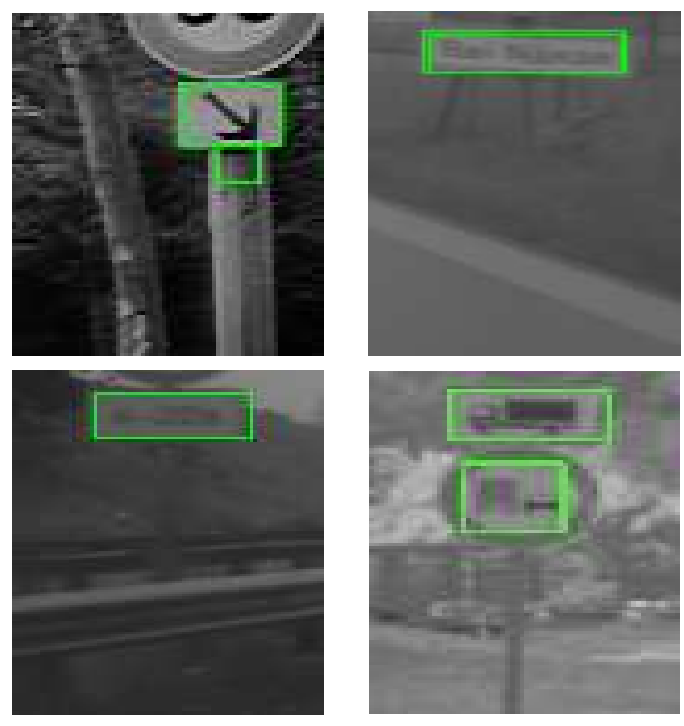

Fig. 5. Some good results obtained with the region-growing method for French and German subsigns. 


\begin{tabular}{|c|c|c|c|c|c|c|c|c|c|}
\hline \multirow{3}{*}{} & \multirow{2}{*}{ Method } & \multicolumn{2}{|c|}{$J \geq 0.5$} & \multicolumn{2}{c|}{$O \geq 0.5$} & \multicolumn{2}{c|}{$O \geq 0.5 \& D \leq 1.5$} & \multicolumn{2}{c|}{$C \leq 0.2$} \\
\cline { 3 - 10 } & & fr & rank & fr & rank & fr & rank & fr & rank \\
\hline \multirow{5}{*}{$d b F$} & Edge & 0.60 & 2 & 0.80 & 3 & 0.73 & 2 & 0.51 & 3 \\
& Color & 0.48 & 3 & 0.73 & 4 & 0.53 & 4 & 0.56 & 2 \\
& Graph & 0.47 & 4 & 0.81 & 2 & 0.58 & 3 & 0.49 & 4 \\
& Region & 0.66 & $\mathbf{1}$ & 0.89 & $\mathbf{1}$ & 0.80 & $\mathbf{1}$ & 0.64 & $\mathbf{1}$ \\
\hline \multirow{5}{*}{$d b G$} & Edge & 0.69 & 2 & 0.85 & 3 & 0.77 & 2 & 0.58 & 2 \\
& Color & 0.40 & 4 & 0.76 & 4 & 0.46 & 4 & 0.45 & 4 \\
& Graph & 0.68 & 3 & 0.90 & $\mathbf{1}$ & 0.72 & 3 & 0.58 & 2 \\
& Region & 0.75 & $\mathbf{1}$ & 0.90 & $\mathbf{1}$ & 0.78 & $\mathbf{1}$ & 0.73 & $\mathbf{1}$ \\
\hline
\end{tabular}

TABLE II

RESULTS OBTAINED ON THE TWO DATABASES FOR THE FOUR IMPLEMENTED TECHNIQUES. NUMBER OF SUBSIGNS CORRECT IN THE SENSE OF EACH CRITERIA PER FRAME AND RANKING.
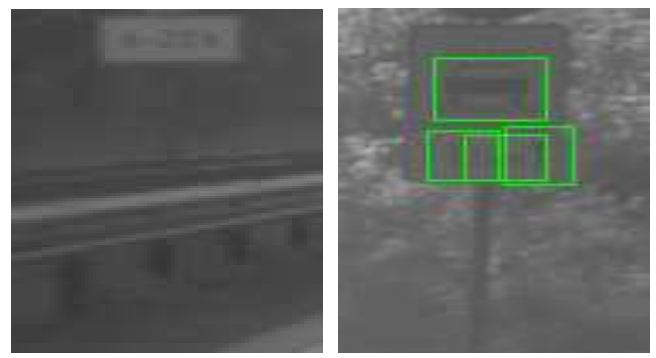

Fig. 6. Some bad results obtained with the region-growing method due to no seed selection (left) or a low contrast (right).

detection above $70 \%$.

As future work, we aim at developing the recognition stage in order to validate the complete process. A research axis is to split the subsigns into different meta categories, for instance text, arrows and vehicle pictograms to perform a first coarse classification. Then a finer recognition will give the final subsign type and eventually the written message. Finally, the complete system has to be tested under real-time and various weather conditions.

\section{ACKNOWLEDGMENTS}

This work was conducted in the framework of the Speedcam project (ANR-09-VTT-11), funded by the ANR (Agence Nationale de la Recherche).

\section{REFERENCES}

[1] O. Hamdoun, A. Bargeton, F. Moutarde, B. Bradai, and L. Chanussot, "Recognition of End-of-Speed-Limit and Supplementary Signs for Improved Speed Limit Support," in World Congress on Intelligent Transport Systems and ITS America's 2008 Annual Meeting, 2008.

[2] W. Liu, J. Lv, H. Gao, B. Duan, H. Yuan, and H. Zhao, "An Efficient Real-Time Speed Limit Signs Recognition Based on Rotation Invariant Features," in IEEE Intelligent Vehicles Symposium. IEEE, 2011, pp. $1000-1005$.

[3] D. Nienhueser, T. Gumpp, J. Zollner, and K. Natroshvili, "Fast and reliable recognition of supplementary traffic signs," in IEEE Intelligent Vehicles Symposium, 2010, pp. 896-901.

[4] C. Jung and R. Schramm, "Rectangle detection based on a windowed hough transform," in Brazilian Symposium on Computer Graphics and Image Processing, 2004, pp. 113-120.

[5] C. Keller, C. Sprunk, C. Bahlmann, J. Giebel, and G. Baratoff, "RealTime Recognition of US Speed Signs," in IEEE Intelligent Vehicles Symposium. IEEE, 2008, pp. 518-523.

[6] S. Chang, L. Chen, Y. Chung, and S. Chen, "Automatic License Plate Recognition," IEEE Transactions on Intelligent Transportation Systems, vol. 5, no. 1, pp. 42-53, 2004.

[7] W. Wu, X. Chen, and J. Yang, "Detection of Text on Road Signs from Video," IEEE Transactions on Intelligent Transportation Systems, [8] vol. 6 , no. 4 , pp. 378-390, 2005.

[9] L. Vincent, "Morphological Grayscale Reconstruction in Image Analysis: Applications and Efficient Algorithms," IEEE Transactions on Image Processing, vol. 2, no. 2, pp. 176-201, 1993.

[10] Z. Zhang, C. Chen, J. Sun, and K. Luk Chan, "E.M. Algorithms for Gaussian Mixtures with Split-and-merge Operation," Pattern Recognition, vol. 36, no. 9, pp. 1973-1983, 2003.

[11] P. Felzenszwalb and D. Huttenlocher, "Efficient Graph-Based Image Segmentation," International Journal of Computer Vision, vol. 59, no. 2, pp. 167-181, 2004. 


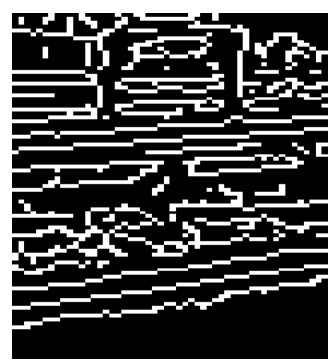

(a) Canny image.

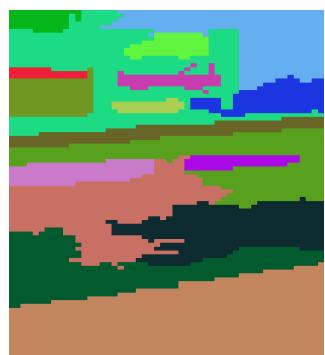

(c) Final regions output by (d) Corresponding rectangraph-based.

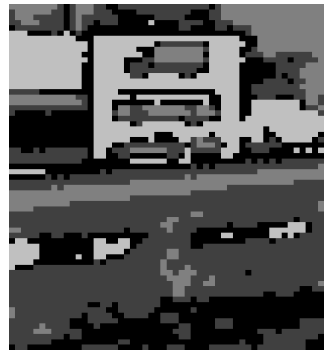

(e) Segmentation obtained from the color-based approach.
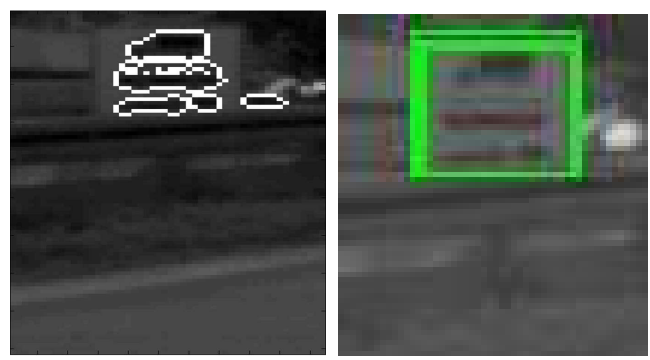

(g) Initial seeds of the re- (h) Output of the region gion growing. growing.

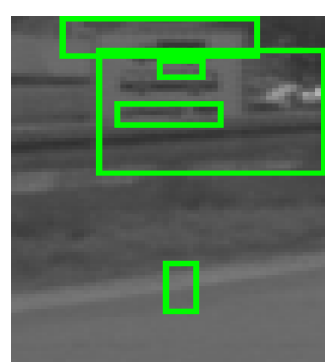

(f) Filtered rectangles.

Fig. 7. Examples of segmentations obtained for the different implemented techniques. 